ORIGINAL STUDY

\title{
The role of ultrasound in the diagnosis of obstructive sleep apnea
}

\author{
Irén Csiszér ${ }^{1,2}$, Silvu Albu ${ }^{1,3}$, Cristian Mircea Neagos4, Marius Navadarszki ${ }^{4}$, Adriana Neagos ${ }^{2,5}$ \\ 1"Iuliu Hatieganu" University of Medicine and Pharmacy, Cluj-Napoca, Romania \\ 2Otorhinolaryngology Department, Galenus Medical Center, Tirgu Mures, Romania \\ 32nd Department of Otorhinolaryngology, "Iuliu Hatieganu" University of Medicine and Pharmacy, \\ Cluj-Napoca, Romania \\ ${ }^{4}$ University of Medicine and Pharmacy of Tirgu Mures, Tirgu Mures, Romania \\ ${ }^{5}$ Otorhinolaryngology Department, University of Medicine and Pharmacy of Tirgu Mures, Tirgu Mures, Romania
}

\begin{abstract}
OBJECTIVE. This study examines the association between the severity of obstructive sleep apnea and the pharyngeal parameters using the ultrasound of the submental region.

MATERIAL AND METHODS. For this study, data obtained from 40 patients, who had undergone investigations in the Galenus Medical Centre in order to diagnose sleep apnea, was analyzed. The following parameters were compared: the transverse diameter of the retrolingual region, the transverse diameter of the retropalatal region and the tongue base thickness. These regions were measured at different moments of respiration such as: during forced expiration, during forced inspiration and while the patients performed Müller's Manoeuvre. The neck circumference, the body mass index of patients and the severity of sleep apnea obtained from polysomnography were analyzed.

RESULTS. The evaluation of the results revealed: severe obstructive sleep apnea in 16 patients, respectively moderate severity in 10 patients, mild apnea in 4 patients, and no obstructive sleep apnea in 10 patients. Statistically significant results $(\mathrm{p}<0.05)$ were found after comparing the parameters obtained in patients with severe apnea versus the parameters in patients without apnea, with mild or respectively moderate apnea.

CONCLUSION. Based on the results obtained, we consider that cervical ultrasound is useful in the diagnosis of severe obstructive apnea, identifying the pathological changes of the anatomical structures that basically cause this category of disease. Due to the fact that in our country ultrasound is a relatively inexpensive method, being also a non-irradiating, repeatable and accessible method, it should be more widely applied also in the field of otorhinolaryngology in order to view structures accessible to this type of imaging.
\end{abstract}

KEYWORDS: ultrasound, obstructive sleep apnea.

\section{INTRODUCTION}

Obstructive sleep apnea syndrome (OSAS) is a common disease in the population. It occurs more frequently in males, at any age, and severely affects the quality of life of the patients who develop the disease $^{1-4}$. Sleep apnea is the pathological condition that is characterized by stopping the airflow for more than 10 seconds during sleep. Depending on the cause of apnea, three types are described in the literature: obstructive apnea, central apnea or mixed apnea.
During sleep, in patients with OSAS, the upper airways collapse and there is only respiratory effort. This collapse is due to changes in the anatomical structure of the airways ${ }^{1,2,5,6}$. These changes have several causes, such as: the increase in volume of the palatine tonsils or the tongue; the decrease in muscle tone of the neck muscles, of the palatine veil or the tongue; the increase in neck circumference, etc. Undiagnosed or untreated on time OSAS seriously affects the quality of life and may lead to the development of severe disease of the cardiovascular and the nervous systems, the appearance of some meta- 


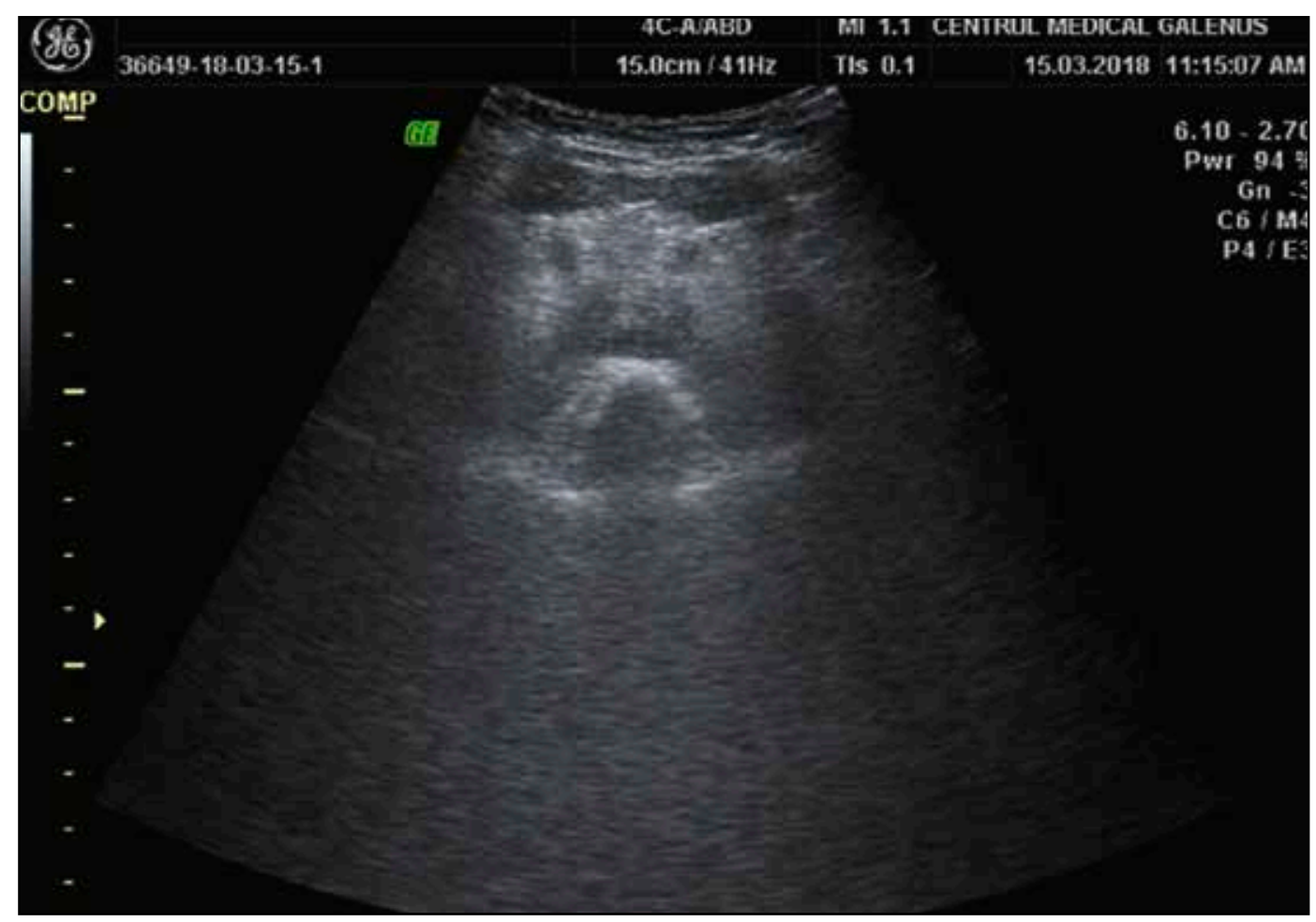

Figure 1 Ultrasonography of the submental region.

bolic diseases, and in some cases can even lead to sudden death ${ }^{1-4,7}$.

In our country, the diagnosis of OSAS is very rare, in most cases being accidentally discovered during a consultation for other disorders.

Based on the apnea-hypopnea index (AHI), the obstructive sleep apnea syndrome is classified in four degrees: none/minimal with $\mathrm{AHI}<5$; of mild severity with AHI $\leq 5$ but less than 15 ; moderate $\geq 15$ and $<30$; sever cu AHI $>30$. AHI is the number of apneas manifested in one hour of sleep.

The gold standard in OSAS diagnosis is polysomnography, but this investigation does not show the anatomic level where the pathological change occurs. The polysomnography must be supplemented by other investigations ${ }^{5-8}$.

This study is retrospective and observational, analyzing the association between the severity of obstructive sleep apnea and the pharyngeal parameters using the ultrasound of the submental region. The study follows the dynamic anatomical changes of the upper respiratory tract using ultrasonography (US).

\section{MATERIAL AND METHODS}

For this study, data obtained from 40 adult patients, who had undergone extensive investiga- tions in the Galenus Medical Centre of Tirgu Mures in order to diagnose sleep apnea, was analyzed. The result of the polysomnography, the body mass index (BMI), the neck circumference, the parameters obtained at the ultrasonography (US) of the submental region (Figure 1) were evaluated.

In order to perform the US, a Voluson 730 PRO V was used, with the convex and linear probe, 2D mode and Color Doppler. The retrolingual and the retropalatal regions were evaluated, measuring their transverse diameter $(\mathrm{mm})$, as well as the tongue base thickness. These parameters were analyzed and compared in patients with varying degrees of severity of OSAS. Measurements were made at different moments of respiration such as: during forced expiration, during forced inspiration and while the patients performed

\section{RESULTS}

Analyzing the results of the polysomnography, severe obstructive sleep apnea was identified in 16 patients, respectively of moderate severity in 10 patients, mild apnea in 4 patients, and no obstructive sleep apnea in 10 patients.

The ultrasound study of the retrolingual and 
the retropalatal regions during inspiration and expiration, as well as of the tongue base thickness, showed statistically significant results ( $\mathrm{t}$ - test, $\mathrm{p}<0.05$ ) after comparing the parameters obtained in patients with severe apnea versus those of patients without apnea, mild apnea and, respectively, moderate apnea (Table 1). The transverse diameter of the retrolingual region, respectively the retropalatal region, showed a decrease in value while increasing the severity of apnea. This trend could be observed both during expiration and during inspiration. If in patients with mild apnea the transverse diameter of the retrolingual space varied between 21 and $32 \mathrm{~mm}$ (a mean of 27.75 ), in the case of a severe syndrome this diameter varied between 11 and $22 \mathrm{~mm}$ (a mean of 16.19). During expiration, the mild form of the sleep apnea syndrome was associated with a mean of the transverse diameter of the retrolingual space of 39 (minimum $38 \mathrm{~mm}$, maximum $40 \mathrm{~mm}$ ), while the severe syndrome can be associated with a diameter of 32 to $36 \mathrm{~mm}$ (a mean of 33.81 ). The same observation can be made in the case of the tongue base thickness, which in the case of a mild sleep apnea syndrome may vary between 39 and $42 \mathrm{~mm}$ (a mean of 40.25), and in the case of a severe syndrome between 46 and $54 \mathrm{~mm}$ (a mean of 49.81).

Moreover, one observed that the values of the retrolingual and the retropalatal regions showed a decrease during inspiration and an increase during expiration, regardless of the apnea severity (Table 1).

The AHI distribution in the group of patients included in the study showed a variation of 1 to 40 apneas / hour, with a mean value of about 20, as can be seen in Figure 2 and Table 2. BMI showed a variation in the study group from 24 to 37, with a mean value of 30.02 , which reveals that most patients had a BMI above normal. The cervical circumference was between 26 and $43 \mathrm{~cm}$, with a mean value of $34.52 \mathrm{~cm}$. A direct proportional correlation between the increase in AHI, BMI and cervical circumference was observed.

Table 1

Variation of Apnea-Hypopnea Index (AHI) depending on the obstruction level and severity. Descriptive Statistics

\begin{tabular}{|c|c|c|c|c|c|}
\hline Parameters & $\mathbf{N}$ & Minimum (mm) & Maximum (mm) & Mean (mm) & Std. Deviation \\
\hline OSAS_severe_RL_i & 16 & 11 & 22 & 16.19 & 3.229 \\
\hline OSAS_moderate_RL_i & 10 & 22 & 30 & 26.20 & 2.201 \\
\hline OSAS_mild_RL_i & 4 & 21 & 32 & 27.75 & 4.787 \\
\hline Normal_RL_i & 10 & 33 & 39 & 35.10 & 2.132 \\
\hline OSAS_severe_RP_i & 16 & 26 & 31 & 28.75 & 1.807 \\
\hline OSAS_moderate_RP_i & 10 & 29 & 37 & 32.70 & 2.263 \\
\hline OSAS_mild_RP_i & 4 & 34 & 38 & 35.75 & 1.708 \\
\hline Normal_RP_i & 10 & 32 & 38 & 35.90 & 2.132 \\
\hline OSAS_severe_Tb & 16 & 46 & 54 & 49.81 & 2.509 \\
\hline OSAS_moderate_Tb & 10 & 42 & 48 & 44.80 & 2.251 \\
\hline OSAS_mild_Tb & 4 & 39 & 42 & 40.25 & 1.258 \\
\hline Normal_Tb & 10 & 38 & 44 & 40.50 & 2.121 \\
\hline OSAS_severe_RL_e & 16 & 32 & 36 & 33.81 & 1.109 \\
\hline OSAS_moderate_RL_e & 10 & 27 & 39 & 36.50 & 3.472 \\
\hline OSAS_mild_RL_e & 4 & 38 & 40 & 39.00 & .816 \\
\hline Normal_RL_e & 10 & 38 & 44 & 41.40 & 2.011 \\
\hline OSAS_severe_RP_e & 15 & 22 & 27 & 25.07 & 1.486 \\
\hline OSAS_moderate_RP_e & 10 & 29 & 35 & 32.40 & 2.459 \\
\hline OSAS_mild_RP_e & 4 & 36 & 42 & 39.00 & 2.582 \\
\hline Normal_RP_e & 10 & 37 & 44 & 40.90 & 2.601 \\
\hline
\end{tabular}

OSAS - obstructive sleep apnea syndrome; $\mathrm{RL} \_\mathrm{i}$ - retrolingual region during inspiration; $\mathrm{RL} \_\mathrm{e}$ - retrolingual region during expiration; $\mathrm{RP} \_\mathrm{i}$ - retropalatal region during inspiration; RP_e - retropalatal region during expiration; $\mathrm{Tb}$ - thickness of the tongue 




Figure 2 Distribution of the Apnea-Hypopnea Index (AHI), the body mass index (BMI) and the neck circumference in the studied group.

\section{DISCUSSIONS}

In the protocol for the evaluation of patients with sleep apnea, both the polysomnography and the ultrasonography of the submental region were included in order to demonstrate the efficiency of the ultrasonography in the diagnosis of the sleep apnea syndrome.

The ultrasonographic measurements targeted the retrolingual region, the retrovelar region and the tongue base thickness. Since many studies have shown that these structures play an important role in the pathogenesis of OSAS, we have tried to focus on a non-invasive, non-irradiating, accessible and efficient method in determining the obstruction site at the oropharyngeal and hy- popharyngeal levels. The ultrasonography is a method that can guide the ENT surgeon in choosing and identifying the anatomical area involved in the pathogenesis of the apnea, in order to obtain a result with maximal postoperative efficiency in the affected patient.

US has the advantage of being able to highlight the structures in dynamics, thus being superior to static imaging methods. It can demonstrate the risk of rapid and repeatable upper airway collapse. The main disadvantage is that it can only measure the transverse retrovelar and retrolingual diameters, not the antero-posterior one. Nevertheless, this aspect is not an obstacle to the applicability of ultrasonography in the case of patients suspected of severe or moderate OSAS, since the transverse di-

Table 2

Distribution of the Apnea-Hypopnea Index (AHI), the body mass index (BMI) and the neck circumference in the studied group.

\begin{tabular}{llcccc}
\hline & N & Minimum & Maximum & Mean & Std. Deviation \\
\hline AHI & 40 & 1 & 40 & 20.17 & 13.597 \\
\hline Neck_circumference & 40 & 26 & 43 & 34.52 & 4.997 \\
\hline BMI & 40 & 24 & 37 & 30.02 & 4.148 \\
\hline Valid N (listwise) & 40 & & & & \\
\hline
\end{tabular}


ameters change in dynamics to a greater extent than the anterior-posterior ones. The sensitivity and specificity of this investigation have been demonstrated in the studies performed on the role of ultrasonography $y^{3,7,8}$.

Due to the fact that in our country ultrasound is a relatively inexpensive method, and taking into account that it is a non-irradiating, repeatable and accessible method, it should be more widely applied also in the field of otorhinolaryngology in order to view structures accessible to this type of imaging.

\section{CONCLUSIONS}

Based on the results obtained, we believe that the cervical ultrasound is useful in the diagnosis, respectively the screening of severe obstructive apnea. It helps identify the pathological changes of the anatomical structures involved in the pathogenesis of this syndrome by allowing the measurement of the pharyngeal parameters, the tongue and the parapharyngeal structures.

Acknowledgments. The authors thank their colleagues in the ENT Department at the Galenus Medical Center of Tirgu Mures who contributed to the evaluation of patients.

Conflict of interest: The authors have no conflict of interest.

Contribution of authors: All authors have equally contributed to this work.

\section{REFERENCES}

1. Lahav Y, Rosenzweig E, Heyman Z, Doljansky J, Green A, Dagan Y. Tongue base ultrasound: a diagnostic tool for predicting obstructive sleep apnea. Ann Otol Rhinol Laryngol. 2009;118(3):179-84.

2. Chen JW, Huang CC, Weng CK, Chang CH, Wang SJ. Simultaneous recording of ultrasound and polysomnography during natural sleep in patients with obstructive sleep apnea: a pilot study. J Sleep Res. 2017;26(4):481-6. DOI: 10.1111/jsr.12515. Epub 2017 Mar 17.

3. Shu CC, Lee P, Lin JW, Huang CT, Chang YC, Yu CJ, et al. The Use of Sub-Mental Ultrasonography for Identifying Patients with Severe Obstructive Sleep Apnea. PLoS One. 2013;8(5):e62848. DOI: 10.1371/journal.pone.0062848.

4. Sólyom R, Csiszér I, Neagos A.Tonsillar hypertrophy implications in sleep disorders in adults and children. Rom J Morphol Embryol. 2014;55 (2 Suppl):603-6.

5. Castellanos-Ramírez JC, Ruíz AJ, Hidalgo-Martínez P, Otero-Mendoza L. Sleep characteristics in an adult with sleep complaints in three cities at different altitudes. J Bras Pneumol. 2018;44(1):65-8. DOI: $10.1590 /$ S1806-37562016000000078.

6. Macey PM, Haris N, Kumar R, Thomas MA, Woo MA, Harper RM. Obstructive sleep apnea and cortical thickness in females and males PLoS One. 2018;13(3):e0193854. DOI: 10.1371/journal. pone.0193854. eCollection 2018.

7. Irani SK, Oliver DR, Movahed R, Kim YI, Thiesen G, Kim KB. Pharyngeal airway evaluation after isolated mandibular setback surgery using cone-beam computed tomography. Am J Orthod Dentofacial Orthop. 2018;153(1):46-53. DOI: 10.1016/j.ajodo.2017.05.031.

8. Castro D, Freeman LA. Airway, Oropharyngeal. [Update 2017 Oct 31]. In: StatPearls [Internet]. Treasure Island (FL): StatPearls Publishing; 2018. Available from: https://www.ncbi.nlm.nih.gov/ books/NBK470198. 